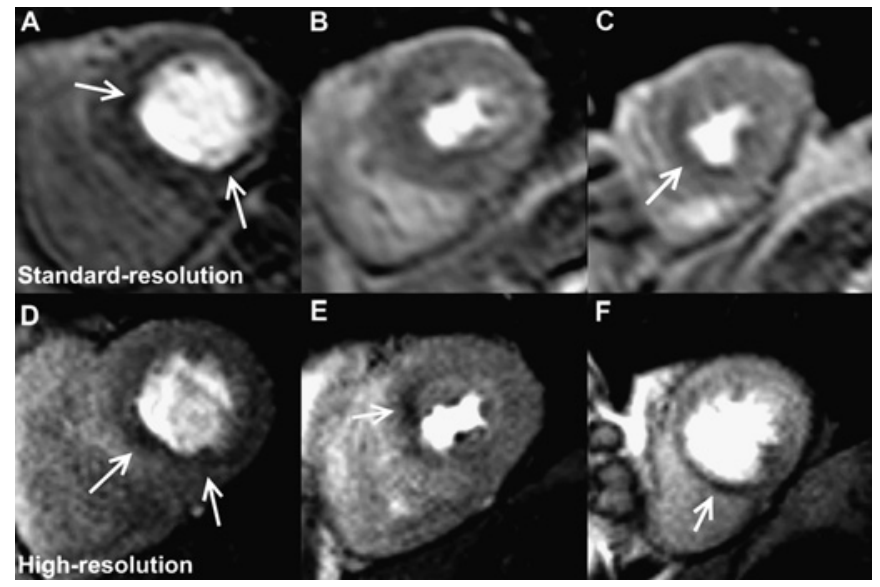

Abstract 087 Figure 1 Case example: Proximal left anterior descending and right coronary artery disease standard-resolution shows possible perfusion defects (white arrows) in the basal anteroseptal, inferoseptal and inferior segments which are difficult to distinguish from dark-rim artifacts $(A)$; and a more convincing perfusion defect in the apical septal segment (C). High-resoultion more clearly delineates perfusion defects in the basal infero-septal, basal inferior (D), mid-anteroseptal (E) and apical septal (F) segments. At high-resloution, the transmural extent of ischaemia can be clearly assessed (the basal and mid-ventricular defects appear subendocardial) and the perfusion defects are better delineated.

resolution acquisition was facilitated by eightfold k-t broad linear speed up technique (BLAST) acceleration. Two observers visually graded perfusion in each myocardial segment on a 4-point scale. Segmental scores were summed to produce a perfusion score for each patient. All patients underwent invasive coronary angiography. Significant $\mathrm{CAD}$ was defined as a coronary artery stenosis of $\geq 50 \%$ diameter on quantitative coronary angiography.

Results CMR data were successfully obtained in 100 patients. A typical example is shown in Abstract 087 figure 1. In patients with CAD $(n=70)$, more segments were determined to have subendocardial ischaemia with high-resolution acquisition than with

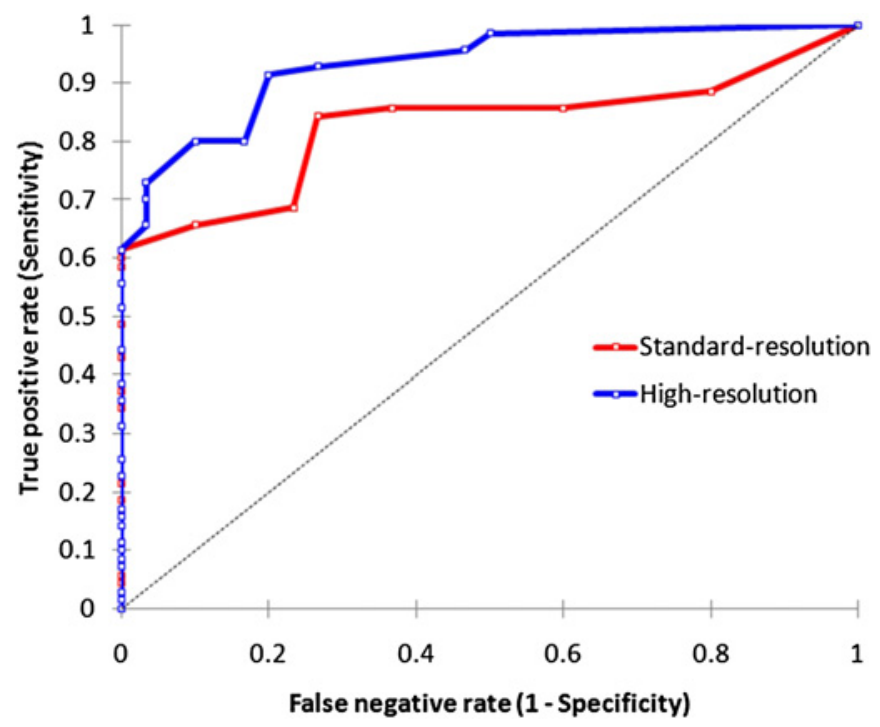

Abstract 087 Figure 2 Receiver-Operator Characteristic Curves. Standard and high-resolution perfusion CMR both had a high diagnostic accuracy for the detection of coronary artery disease but the highresolution technique was superior. The arears under the curve were 0.83 (95\% Cl 0.75 to 0.91$)$ for standard-resolution and 0.93 (95\% Cl 0.88 to $0.98)$ for high-resolution $(p<0.001)$. standard-resolution acquisition (279 vs 108; $\mathrm{p}<0.001$ ). High-resolution acquisition had a greater diagnostic accuracy than standardresolution acquisition for identifying single-vessel disease (area under the curve [AUC]: 0.88 vs $0.73 ; \mathrm{p}<0.001)$ or multi-vessel disease (AUC: 0.98 vs $0.91 ; p=0.002$ ) and overall (AUC: 0.93 vs 0.83 ; $\mathrm{p}<0.001$ ) (Abstract 087 figure 2).

Conclusions Our study shows that high-resolution CMR perfusion imaging has greater diagnostic accuracy than standard-resolution acquisition for the detection of $\mathrm{CAD}$ in both single and multi-vessel disease patients and detects more subendocardial ischaemia.

\section{COMPARISON OF CARDIOVASCULAR MAGNETIC RESONANCE STRESS PERFUSION WITH SINGLE PHOTON EMISSION CT (SPECT) IN PATIENTS WITH LEFT MAIN STEM DISEASE: A CE-MARC SUBSTUDY}

doi:10.1136/heartjnl-2012-301877b.88

${ }^{1} \mathrm{~J}$ P Greenwood, ${ }^{1} \mathrm{~A}$ Kidambi, ${ }^{1} \mathrm{~A}$ Zaman, ${ }^{1} \mathrm{~N}$ Maredia, ${ }^{1} \mathrm{M}$ Motwani, ${ }^{2} \mathrm{C}$ J Dickinson, ${ }^{3} \mathrm{~J}$ M Brown, ${ }^{3} \mathrm{~J}$ Nixon, ${ }^{3} \mathrm{C}$ C Everett, ${ }^{1} \mathrm{~S}$ G Ball, ${ }^{1} \mathrm{~S}$ Plein. ${ }^{1}$ Multidisciplinary Cardiovascular Research Centre \& Leeds Institute of Genetics, Health and Therapeutics, University of Leeds, UK; ${ }^{2}$ Leeds Teaching Hospitals NHS Trust, Leeds, UK; ${ }^{3}$ University of Leeds, Leeds, UK

Introduction Approximately 5\% of patients with stable angina have disease of the left main stem (LMS). Three-year survival in patients with $>50 \%$ LMS stenosis may be as low as $50 \%$. Single photon emission tomography (SPECT) fails to detect ischaemia in up to $15 \%$ of LMS stenoses, and identification of the "classical" pattern of both left anterior descending (LAD) and circumflex (LCx) coronary territory ischaemia is lower still. To date, the utility of cardiovascular magnetic resonance (CMR) perfusion in LMS disease is poorly established. The CE-MARC study was a prospective study of 752 patients with suspected coronary artery disease, enrolled to undergo CMR, SPECT and x-ray coronary angiography; we assessed the diagnostic performance of SPECT and CMR to detect LMS disease. Methods All patients with LMS disease $\geq 50 \%$ on quantitative angiography were identified from the CE-MARC study. All patients had undergone adenosine stress perfusion by CMR and SPECT and also invasive $\mathrm{x}$-ray angiography. ${ }^{1}$ We compared detection rates for visual perfusion analysis from both the CMR and SPECT perfusion studies in patients with $\geq 50 \%$ and $\geq 70 \%$ LMS stenosis on angiography.

Results Of 23 patients in the CE-MARC cohort with LMS stenosis $\geq 50 \%$, one patient could not be analysed. CMR identified evidence

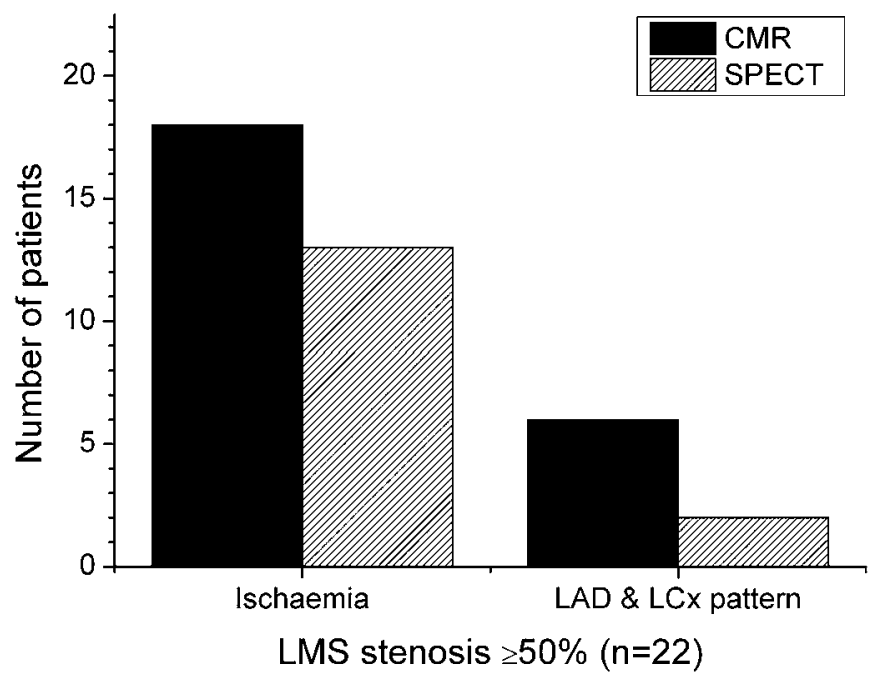

Abstract 088 Figure 1 Detection rate of CMR and SPECT in patients with $\geq 50 \%$ LMS stenosis. 


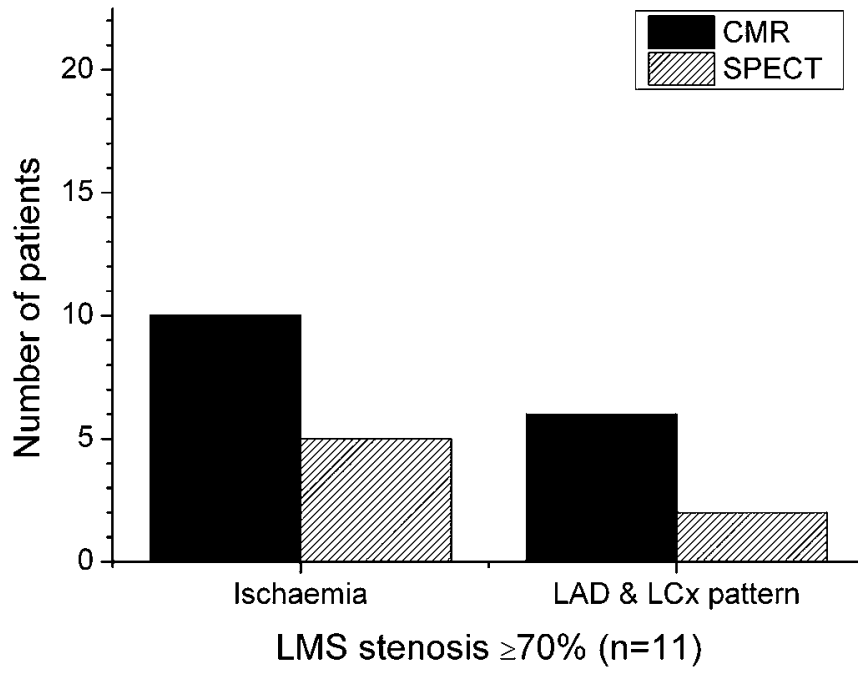

Abstract 088 Figure 2 Detection rate of CMR and SPECT in patients with $\geq 70 \%$ LMS stenosis.

of inducible perfusion defects in 18/22 (82\%) of the LMS group; SPECT identified 13/22 (59\%). For CMR and SPECT respectively, inducible perfusion defects were found in both LAD and LCX territories for $6 / 18(33 \%)$ and $2 / 13(15 \%)$. Only one patient had normal perfusion analyses (false negative) for both CMR and SPECT. Of 11 patients with $\geq 70 \%$ LMS stenosis, 10 (91\%) had inducible perfusion defects with CMR vs $5(45 \%)$ with SPECT. Six $(55 \%)$ vs $2(18 \%)$ had a LAD and LCx disease pattern. Perfusion abnormalities were detected with similar frequency in $\geq 50 \%$ and $\geq 70 \%$ groups by both CMR $(p=0.64)$ and SPECT $(p=0.49)$. Abstract 088 figures 1 and 2 summarise the detection rate of CMR and SPECT in LMS disease in the $\geq 50 \%$ and $\geq 70 \%$ LMS groups respectively.

Conclusions CMR stress perfusion imaging identifies ischaemia in a higher proportion of patients with significant LMS disease than SPECT, and identifies a "classical" LMS pattern with higher frequency. Perfusion abnormalities are detected with similar frequency in patients with $\geq 50 \%$ and $\geq 70 \%$ LMS stenosis.

\section{REFERENCE}

1. Greenwood JP, et al. CE-MARC: a prospective evaluation of cardiovascular magnetic resonance and single-photon emission computed tomography in coronary heart disease. Lancet. In Press.

\section{REGRESSION OF MYOCARDIAL OEDEMA IS RELATED TO IMPROVEMENT IN MYOCARDIAL CONTRACTILITY FOLLOWING REPERFUSED ACUTE MYOCARDIAL INFARCTION}

\section{doi:10.1136/heartjnl-2012-301877b.89}

A Kidambi, ${ }^{*}$ A N Mather, P Swoboda, M Motwani, J P Greenwood, S Plein. Multidisciplinary Cardiovascular Research Centre \& Leeds Institute of Genetics, Health and Therapeutics, University of Leeds, Leeds, UK

Introduction Myocardial oedema is a feature of reperfused acute myocardial infarction (AMI), and contributes to stunning of periinfarct myocardium (the "area at risk"). Regression of oedema on T2 weighted (T2w) cardiovascular magnetic resonance (CMR) imaging is related to improved myocardial contractility post AMI in animal models, but has not been established in man. We hypothesised that resolution of tissue oedema correlates with recovery of regional contractile function.

Methods Patients after primary percutaneous coronary intervention for first ST-elevation AMI underwent CMR with $\mathrm{T} 2 \mathrm{w}$ imaging, myocardial tagging and late gadolinium enhancement at 2, 30 and 90 days following reperfusion. Infarct size, regional circumferential strain, T2w signal intensity and volume of myocardial oedema were measured for infarct zone, peri-infarct zone and remote myocardium. Oedema and infarction were defined as zones with signal intensity 2 SDs above remote myocardium in T2w and LGE imaging respectively. $\mathrm{T} 2 \mathrm{w}$ signal intensity was normalised to remote myocardium.

Results 30 patients had CMR imaging at all 3 time points with adequate image quality and sufficient peri-infarct oedema for quantitative analysis. Circumferential strain was significantly diminished in infarct and peri-infarct zones compared to remote myocardium (means -0.149 vs -0.184 vs $-0.236, p<0.01$ between groups and $\mathrm{p}<0.01$ for trend). Remote myocardium showed no significant change in strain over time $(\mathrm{F}=1.44, \mathrm{p}=0.24)$, while the peri-infarct zone $(\mathrm{F}=6.03, \mathrm{p}=0.004)$ and infarct zone $(\mathrm{F}=20.34$, $\mathrm{p}<0.001$ ) showed a significant increase in magnitude (Abstract 089 table 1). This change closely mirrored resolution of both intensity and volume of $\mathrm{T} 2 \mathrm{w}$ hyperenhancement (Abstract 089 figure 1). Decreased circumferential strain correlated significantly with T2w volume $(r=0.30 ; p<0.01)$ and normalised $T 2 w$ signal intensity $(r=0.28 ; p<0.01)$. Due to the finding of recovery of function in the infarct zone, we analysed 8 patients with complete transmural infarction. The area of fully transmural infarction showed significant resolution of strain with time (means -0.103 (day 2), -0.148 (day 30) and -0.194 (day 90); $\mathrm{p}=0.04$ for trend).

Abstract 089 Table 1 Mean circumferential strain by myocardial location and time

\begin{tabular}{llll}
\hline & Infarct zone & Peri-infarct zone & Remote zone \\
\hline 2 days & -0.102 & -0.137 & -0.226 \\
30 days & -0.160 & -0.188 & -0.240 \\
90 days & -0.186 & -0.207 & -0.241 \\
2 days-30 days comparison & $(<0.001)$ & $(0.01)$ & $(0.10)$ \\
2 days-90 days comparison & $(<0.001)$ & $(0.002)$ & $(0.17)$ \\
30 days-90 days comparison & $(0.04)$ & $(0.14)$ & $(1.00)$ \\
\hline
\end{tabular}

$\mathrm{p}$ Values in parentheses.

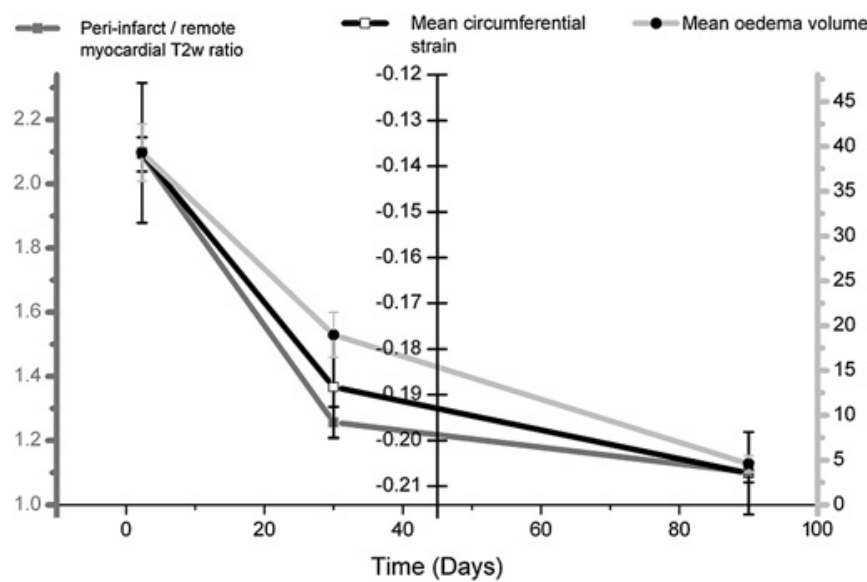

Abstract 089 Figure 1 Change in strain, T2w signal intensity, and oedema volume in the peri-infarct zone.

Conclusions Early after AMI, oedematous myocardium in the periinfarct zone demonstrated significantly reduced strain as compared to remote myocardium. Improvement of strain in stunned myocardium closely followed the regression of myocardial oedema. Patients with larger oedema volumes and higher signal intensity on T2w imaging demonstrated greater improvement of strain within the area at risk. In addition, both transmural and subendocardial 